\title{
On the Category of Ideals of Ring
}

\author{
P. G. Romeo ${ }^{1}$ and Sreejamol P. R. ${ }^{2}$ \\ 1. Professor, Dept. of Mathematics, Cochin University of Science and Technology, Kochi, Kerala, India. \\ 2. Assistant Professor, Department of Mathematics, SNM College, Maliankara, Ernakulam, Kerala, India.
}

Received: December 06, 2016 / Accepted: January 03, 2017 / Published: March 25, 2017.

\begin{abstract}
In this paper we describe the category whose objects are principal ideals of a ring and morphisms are appropriate translations and it is shown that such a category is an abelian category. Further we discuss various properties of such categories.
\end{abstract}

Keywords: Ring, Ideals of ring, Category of ideals of ring, Abelian category.

\section{Introduction}

There are many successful attempts to use category theory to study several mathematical structures like groups, semigroups, rings etc. The ESN theorem (Erasmann-Schein-Nambooripad theorem) and inverse categories introduced by Lawson (cf.[6]) and the cross-connection categories introduced by Grillet and Nambooripad are remarkable achievements in this direction. In this paper we propose to extend the category theoretical approach to the study of the structure theory of rings. In this regard wedescribe a category whose objects are certain ideals of a ring with morphisms appropriate translations and discuss various properties of such categories.

\section{Preliminaries}

In this section we briefly recall all basic definitions and results regarding categories needed in the sequel, however for any notations or results not explicitly stated the reader is referred to S. Maclane (cf.[1]). A category is an algebra consisting of objects and morphisms that are closed under composition and satisfy certain conditions of the composition of morphisms.

Corresponding author: P. G. Romeo, Professor, Dept. of Mathematics, Cochin University of Science and Technology, Kochi, Kerala, India. E-mail: romeo_parackal@yahoo.com.
Definition 1. A category $C$ consists of object set $\{A, B, C, \ldots\}$ and morphisms (arrows) $\{f, g, h, \ldots\}$ such that for each arrow $f$ there are objects $\operatorname{dom}(f), \operatorname{cod}(f)$ called the domain and codomain of $f$. Given arrows $f$ and $g$, with $\operatorname{cod}(f)=\operatorname{dom}(g)$ then there exists an arrow $g \cdot f$ called the composite of $f$ and $g$ and for each object $A$ is an arrow $I_{A}: A \rightarrow A$ called the identity arrow. Further $C$ satisfying the following

$$
h \cdot(g \cdot f)=(h \cdot g) \cdot f
$$

for composible arrows $f, g, h$

$$
f \cdot I_{A}=f=I_{B} \cdot f, \forall f: A \rightarrow B .
$$

Set, Grp are familiar examples of categories whose objects are sets and morphisms are set maps and the category whose objects are groups and morphisms group homomorphisms respectively. Let $A, B$ be objects in a category $C$, then the set consists of all morphisms of the category with domain $A$ and codomain $B$ is called the hom-set and is denoted as $\operatorname{hom}_{C}(A ; B)$ or $\operatorname{hom}(A ; B)$, ie.,

$$
\operatorname{hom}(A ; B)=\{f: A \rightarrow B \in C\}
$$

Any arrow $g: B \rightarrow B^{\prime}$ in $C$ induces a map

$$
\operatorname{hom}(A ; g): \operatorname{hom}(A ; B) \rightarrow \operatorname{hom}\left(A ; B^{\prime}\right)
$$

defined by 


$$
f \mapsto g \circ f \text { where } f \in \operatorname{hom}(A ; B) \text {. }
$$
A
(covariant)functor
$F: \mathcal{C} \rightarrow \mathcal{D}$
between

categories $\mathcal{C}$ and $\mathcal{D}$ is a mapping of objects to objects and arrows to arrows such that if $f: c \rightarrow c^{\prime} \in \mathcal{C}$ then $F(f): F(c) \rightarrow F\left(c^{\prime}\right)$ and $F\left(I_{c}\right)=I_{F(c)}, \quad F(g \circ f)=F(g) \circ F(f)$. By a functor always means a covariant functor. A (covariant) functor $F: \mathcal{C}^{o p} \rightarrow \mathcal{D}$ is called a contravariant functor of $\mathcal{C}$ to $\mathcal{D}$.

Example 1. Consider $P:$ Set $\rightarrow$ Set which assigns to each set $X$ the usual power set $P(X)$, of all subsets $S \subset X$; its arrow function assigns to each $f: X \rightarrow Y$ a map $P(f): P(X) \rightarrow P(Y)$ which sends each $S \subset X$ to its image $f S \subset Y$. Since $\quad P\left(I_{X}\right)=I_{P(X)} \quad$ and $\quad P(g \circ f)=$ $P(g) \circ P(f)$, this defines a functor $P:$ Set $\rightarrow$ Set known as the power set functor.

Given functors $T: \mathcal{C} \rightarrow \mathcal{B}$ and $S: \mathcal{B} \rightarrow \mathcal{A}$, then the composite function $S \circ T: \mathcal{C} \rightarrow \mathcal{A}$, called the composite of $S$ with $T$.

Example 2. Each object $A \in \mathcal{C}$ defines a functor called hom functor $\operatorname{hom}(A ;-): \mathcal{C} \rightarrow \boldsymbol{S e t}$. This is called the representable functor of $A$ (or the covariant hom-functor determined by A). Clearly

$$
\operatorname{hom}\left(A ; I_{X}\right)=I_{\operatorname{hom}(A ; X)}
$$

and

$$
\operatorname{hom}(A ; g \circ f)=\operatorname{hom}(A ; g) \circ \operatorname{hom}(A ; f)
$$

A functor $T: \mathcal{C} \rightarrow \mathcal{D}$ is an isomorphism if and only if there is a functor $S: \mathcal{D} \rightarrow \mathcal{C}$ for which both composites $S \circ T$ and $T \circ S$ are identity functors; then $S$ is the two sided inverse $S=T^{-1}$.

Definition 2. Given two functors $S, T: \mathcal{C} \rightarrow \mathcal{B}$, a natural transformation $\tau: S \rightarrow T$ is a function which assigns to each object $c$ of $\mathcal{C}$ an arrow $\tau_{c}: S c \rightarrow T c$ of $\mathcal{B}$ in such a way that every $f: c \rightarrow c^{\prime}$ in $\mathcal{C}$ yields $T f \circ \tau_{c}=\tau_{c^{\prime}} \circ S f$. A natural transformation is often called a morphism of functors; ie in which objects are functors and morphisms are natural transformations.

$\mathcal{C}$ and $\mathcal{D}$ be two categories then $[\mathcal{C} ; \mathcal{D}]$ is the category whose objects are functors from $\mathcal{C}$ to $\mathcal{D}$ and morphisms are natural transformations. If $S$ and $T$ are functors, the hom-set of thiscategory is

$$
\operatorname{Nat}(S ; T)=\{\eta / \eta: S \rightarrow T ; \text { natural }\}
$$

and the composition in this category is the component-wise product of natural transformations, that is $\eta \in \operatorname{Nat}(S ; T)$ and $\gamma \in \operatorname{Nat}(T ; U), \eta \gamma$ is the natural transformation defined by

$$
(\eta \gamma)_{c}=\eta_{c} \gamma_{c}
$$

for all $c \in \mathcal{V}$. Any subcategory of $[\mathcal{C} ; \mathcal{D}]$ will be called a functor category from $\mathcal{C}$ to $\mathcal{D}$ and $\mathcal{C}^{*}$ denote the functor category $[\mathcal{C} ;$ Set $]$.

Duality is a correspondence between properties of category $\mathcal{C}$ and dual properties of the opposite category $\mathcal{C}^{o p}$. Given a statement regarding the category $\mathcal{C}$, by interchanging the source and target of each morphism as well as changing the order of composition the dual statement is obtained regarding the opposite category $\mathcal{C}^{o p}$. If a statement is true about $\mathcal{C}$, then its dual statement is true about $\mathcal{C}^{o p}$.

Definition 3. A morphism $f$ in a category $\mathcal{C}$ is a monomorphism if for $g, h \in \mathcal{C}, g f=h f$ implies $g=h$; that is $f$ is a monomorphism if it is right cancelable.

Dually a morphism $f \in \mathcal{C}$ is an epimorphism if $f$ is left cancelable. An object $A$ is terminal in $\mathcal{C}$ if for each object $B$ there is exactly one arrow $B \rightarrow A$. An object $C$ is initial object if to each object $B$ there is exactly one arrow $C \rightarrow B$. A zero object or null object $Z$ in $\mathcal{C}$ is an object which is both initial and terminal. For any two objects $A$ and $B$ the unique arrows $A \rightarrow Z$ and $Z \rightarrow B$ have a composite $O_{B}^{A}: A \rightarrow B$ called the zero arrow from $A \rightarrow B$.

Definition 4. An equalizer of morphisms 
$f, g: B \rightarrow A$ in $C$ is an arrow $e: D \rightarrow B$ such that $f \cdot e=g \cdot e$, to any $h: C \rightarrow B$ with $f \cdot h=g \cdot h$ there is a unique $h^{\prime}: C \rightarrow D$ with $e \cdot h^{\prime}=h$.

Dually coequalizer of $f, g: A \rightarrow B$ is an arrow $u: B \rightarrow F \quad$ such that $u \cdot f=u \cdot g \quad ; \quad$ and if $h: B \rightarrow C$ has $h \cdot f=h \cdot g$, then $h=h^{\prime} \cdot u$ for a unique arrow $h^{\prime}: F \rightarrow C$.

Let $C$ has a zero object. A kernel of an arrow $f: A \rightarrow B$ is defined to be an equalizer of the arrows $f, O: A B$. Dually cokernel of $f: A \rightarrow B$ is an arrow $u: B \rightarrow E$ such that $u f=O: A \rightarrow E$ and if $h: B \rightarrow C$ has $h f=O$, then $h=h^{\prime} u$ for a unique arrow $h^{\prime}: E \rightarrow C$.

Remark 1. A kernel is neccessarily a monomorphism and a cokernel is an epimorphism.

Definition 5. The product of two objects $A$ and $B$ of category $\mathcal{C}$ is written $A \times B$ or $A \Pi B$ with two arrows $p_{1}: A \Pi B \rightarrow A, p_{2}: A \Pi B \rightarrow B$ called the projections of the product $A \Pi B$ such that for any $X \in \mathcal{C}$ with given arrows $f: X \rightarrow A$ and $g: X \rightarrow B$, there is a unique $h: X \rightarrow A \times B$ with $p_{1} \cdot h=f$ and $p_{2} \cdot h=g$.

Dually coproduct as $A+B$ or $A \amalg B$ with two arrows $\quad q_{1}: A \rightarrow A \amalg B, q_{2}: B \rightarrow A \amalg B \quad$ called the injections of the coproduct $A \amalg B$ such that for any $X \in \mathcal{C}$ with given arrows $f: A \rightarrow X$ and $g: B \rightarrow X$, there is a unique $h: A \amalg B \rightarrow X$ with $h \cdot q_{1}=f$ and $h \cdot q_{2}=g$.

Biproduct of a finite collection of objects, in a category with zero objects, is both a product and a coproduct.

An Ab-category $A$ (cf.[1])is a category in which each hom-set $\operatorname{hom}(B ; C)$ is an additive abelian group and composition of arrows is bilinear relative to this addition.
The following proposition is from [1].

Proposition 1. The following properties of an object $Z$ in an Ab-category $A$ are equivalent:

(1) $Z$ is initial;

(2) $Z$ is terminal;

(3) $I_{Z}=O: Z \rightarrow Z$;

(4) the abelian group $\operatorname{hom}(Z, Z)$ is the zero group.

In particular, any initial(or any terminal) object in $A$ is a null object.

Definition 6. An additive category is an Ab-category which has a zero object $O$ and a biproduct for each pair of its objects.

Definition 7. Abelian category $A$ is an Ab-category satisfying the following:

(1) $A$ has a null object,

(2) $A$ has binary products and coproducts,

(3) Every arrow in $A$ has a kernel and a cokernel,

(4) Every monic arrow is a kernel, and every epi a cokernel.

Let $R$ be a ring with unit $I_{R}$. A leftR-module is an abelian group $(M,+)$ together with an operation. from $R \times M$ such that for all $r, s \in R$ and $x, y \in M$

(1) $r \cdot(x+y)=r \cdot x+r \cdot y$

(2) $(r+s) \cdot x=r \cdot x+s \cdot x$

(3) $(r s) \cdot x=r \cdot(s \cdot x)$

(4) $I_{R} \cdot x=x$.

Dually we can define right $R$-module.

Example 3. Every abelian group is a module over the ring of integers.

If $M$ and $N$ are two left $R$-modules, a map $f: M \rightarrow N$ is a homomorphism of $R$-modules if, for any $m, n$ in $M$ and $r, s$ in $R$,

$$
f(r \cdot m+s \cdot n)=r \cdot f(m)+s \cdot f(n)
$$


Category of left $R$-modules as objects and morphisms such module homomorphisms is an abelian category.

Definition 8. Let $R$ be a ring, a nonempty subset $I$ of $R$ is called right ideal of $R$ if

(1) $a \in I$ then $-a \in I$

(2) $a, b \in I$ then $a+b \in I$

(3) $a \in I$, and $r \in R$ then $a r \in I$.

$a \in I$, and $r \in R$ if $r a \in I$ then $I$ is called left ideal of ring $R$ and if both $a r$ and $r a \in I$, then $I$ is called ideal. An ideal generated by a single element $a$ of $R$ is called principal ideal of $R$.

The sum of two ideals $I$ and $J$ of a ring $R$ is

$$
I+J=\{a+b: a \in I ; b \in J\} .
$$

It is easy to see that $I+J$ is an ideal of $R$ and is the smallest ideal of $R$ which contains both $I$ and $J$. Note that a left ideal $I$ in $R$ is a left module over $R$ and a right ideal is a right module.

A pre order $P$ is a category such that, for any $p, p^{\prime} \in v P$, the hom-set $P\left(p, p^{\prime}\right)$ contains at most one morphism. In this case the relation $\subseteq$ on the class $v P$ of objects of $l P$ defined by $p \subseteq p^{\prime} \Leftrightarrow P\left(p, p^{\prime}\right) \neq \phi$ is a quasi-order. $P$ is said to be a strictpreorder if $\subseteq$ is a partial order.

Definition 9. Let $\mathcal{C}$ be a category and $P$ be a subcategory of $\mathcal{C}$. Then $(\mathcal{C}, P)$ is called a category with subobjects if the following hold:

(1) $P$ is a strict pre order with $v P=v \mathcal{C}$.

(2) Every $f \in P$ is a monomorphism in $\mathcal{C}$.

(3) If $f, g \in P$ and if $f=h g$ for some $h \in \mathcal{C}$, then $h \in P$.

In a category with subobjects, if $f: c \rightarrow d$ is a morphism in $P$, then $f$ is said to be an inclusion, we denote this inclusion by $j(c, d)$. Subobjects in a category is an equivalence class of monomorphisms.

A morphism $f \in \mathcal{C}$ with subobjects has a canonical factorization if $f=m \cdot e$ where $m$ is monomorphism and $e$ is an epimorphism.

\section{Ideal Categories}

Let $R$ be a ring and $a \in R$, let $(a)_{r}$ and $(a)_{l}$ denote the principal right ideal and the principal left ideal generated by $a$.

Lemma 1. Let $R$ be a ring, $a \in R$ then $(a)_{r}=\mathbb{Z} a+a R$ and $(a)_{l}=\mathbb{Z} a+R a$; where $\mathbb{Z}$ is the ring of integers. If $1 \in R,(a)_{r}=a R$ and $(a)_{l}=R a$.

Proof. $\mathbb{Z} a+a R=\{n a+a r, n \in \mathbb{Z}, r \in R\}, \quad$ for $x, y \in \mathbb{Z} a+a R, x+y \in \mathbb{Z} a+a R, \quad$ if $x \in \mathbb{Z} a+a R,-x \in \mathbb{Z} a+a R$ and for $s \in R$, $x_{s} \in \mathbb{Z} a+a R$, hence $\mathbb{Z} a+a R$ is the principal right ideal generated by $a$. Similarly $\mathbb{Z} a+R a=(a)_{l}$ is the principal left ideal generated by $a$ in $R$. If $1 \in R$, then $(a)_{r}=a R$ and $(a)_{l}=R a$.

Theorem 1. Let $R$ be a ring, then $\mathscr{P}_{R}$ the category whose objects are principal right ideals of $R$ and hom-set

$$
\begin{aligned}
& \operatorname{hom}\left((a)_{r},(b)_{r}\right)= \\
& \left\{\lambda_{(n, r)}: x \rightarrow n x+r x, n \in Z ; r \in R\right\}
\end{aligned}
$$

is an abelian category.

Proof. For each object $(a)_{r} \in \mathscr{R}_{R}$ there exists a distinguished morphism $i d_{(a)_{r}} \in \operatorname{hom}\left((a)_{r},(a)_{r}\right)$ given by $\lambda_{(1,0)}: x \rightarrow x, \forall x \in(a)_{r} \quad$. Let $\lambda_{(n, r)} \in \operatorname{hom}\left((a)_{r},(b)_{r}\right) \quad$ and $\lambda_{(m, s)} \in h o m\left((b)_{r},(c)_{r}\right) \quad$ for $\quad n, m \in \mathbb{Z} ; \quad r, s \in R$ then morphism $\lambda_{(m, s)} \cdot \lambda_{(n, r)}=\lambda_{(m n, m r+s n+s r)} \in \operatorname{hom}\left((a)_{r},(c)_{r}\right) \quad$ is 
the composite of $\lambda_{(n, r)}$ and $\lambda_{(m, s)}$, also $i d_{(b)_{r}} \cdot \lambda_{(n, r)}=\lambda_{(n, r)}=\lambda_{(n, r)} \cdot i d_{(a)_{r}}$. $\quad$ If for $n, m, k \in \mathbb{Z} ; r, s, t \in R, \lambda_{(n, r)}, \lambda_{(m, s)}$, and $\lambda_{(k, t)}$ are composable morphisms then

$$
\lambda_{(k, t)} \cdot\left(\lambda_{(m, s)} \cdot \lambda_{(n, r)}\right)=\left(\lambda_{(k, t)} \cdot \lambda_{(m, s)}\right) \cdot \lambda_{(n, r)} \cdot
$$

Thus $\mathscr{T}_{R}$ is a category. For $\lambda_{(n, r)}, \lambda_{(m, s)} \in \operatorname{hom}\left((a)_{r},(b)_{r}\right)$, the addition defined by $\lambda_{(n, r)}+\lambda_{(m, s)}=\lambda_{(n+m, r+s)} \in \operatorname{hom}\left((a)_{r},(b)_{r}\right)$ is associative and commutative. $\lambda_{(o, 0)}$ is the additive identity where 0 is the additive identity in $(b)_{r}$, $o \in \in Z$ and $\lambda_{(-n,-r)}$ is the additive inverse of $\lambda_{(n, r)}$ thus $\operatorname{hom}\left((a)_{r},(b)_{r}\right)$ is an abelian group under addition, further if restricted to hom $\left((a)_{r},(a)_{r}\right)$, it is a ring.

For $\quad x, y \in(a)_{r} \quad$ and $\quad r_{1}, r_{2} \in R \quad$, $\lambda_{(n, r)}\left(x r_{1}+y r_{2}\right)=\lambda_{(n, r)}(x) r_{1}+\lambda_{(n, r)}(y) r_{2} \quad, \quad$ thus $\lambda_{(n, r)}$ is a module homomorphism and the ideal generated by the zero element of the ring is the initial objectas well as the terminal object and hence the zero object.

For $\lambda_{\left(n_{1}, r_{1}\right)}, \lambda_{\left(n_{2}, r_{2}\right)} \in \operatorname{hom}\left((b)_{r},(c)_{r}\right)$ and for $\lambda_{(m, s)} \in \operatorname{hom}\left((a)_{r},(b)_{r}\right), \quad \lambda_{(k, t)} \in \operatorname{hom}\left((c)_{r},(d)_{r}\right)$, $\left(\lambda_{\left(n_{1}, r_{1}\right)}+\lambda_{\left(n_{2}, r_{2}\right)}\right) \cdot \lambda_{(m, s)}(x)=$ $\lambda_{\left(n_{1}+n_{2}, r_{1}+r_{2}\right)} \cdot \lambda_{(m, s)}(x)=$ $\lambda_{\left(\left(n_{1}+n_{2}\right) m,\left(n_{1}+n_{2}\right) s+\left(r_{1}+r_{2}\right) m+\left(r_{1}+r_{2}\right) s\right)}(x)=$ $\lambda_{\left(n_{1}, r_{1}\right)} \cdot \lambda_{(m, s)}(x)+\lambda_{\left(n_{2}, r_{2}\right)} \cdot \lambda_{(m, s)}(x)$, for $x \in(a)_{r}$.

Similarly,

$$
\lambda_{(k, t)} \cdot\left(\lambda_{\left(n_{1}, r_{1}\right)}+\lambda_{\left(n_{2}, r_{2}\right)}\right)(x)=
$$

$$
\left(\lambda_{(k, t)} \cdot \lambda_{\left(n_{1}, r_{1}\right)}(x)+\left(\lambda_{(k, t)} \cdot \lambda_{\left(n_{2}, r_{2}\right)}(x),\right.\right.
$$

thus the composition of morphisms in the category $\mathscr{T}_{R}$ is bilinear, thus the category $\mathscr{T}_{R}$ is an Ab-category. For $(a)_{r},(b)_{r} \in \mathscr{R}_{R}$, define

$$
(a)_{r}+(b)_{r}=\left\{\begin{array}{l}
(c)_{r}, \text { if exists } \\
R, \text { otherwise }
\end{array}\right.
$$

where $(c)_{r}$ is the smallest principal ideal containing both $(a)_{r}$ and $(b)_{r}$. It is easy to see that $(a)_{r}+(b)_{r}$ is the product and also the coproduct. Thus $\mathscr{R}_{R}$ is an additive category.

Every arrow $\lambda_{(n, r)}:(a)_{r} \rightarrow(b)_{r}$ given by $x \rightarrow n x+r x, n \in Z ; r \in R$ in $\mathscr{T}_{R}$ has a kernel $k$ as an equalizer of the arrows $\left.\lambda_{(n, r)}, \lambda_{(} o, 0\right):(a)_{r}(b)_{r}$. $k:(O)_{r} \operatorname{ightarrow}(a)_{r}$ is a kernel of $\lambda_{(n, r)}:(a)_{r} \rightarrow(b)_{r}$ when $\lambda_{(n, r)} \cdot k=O$. The kernel $k$ is a monomorphism. Dually cokernel exists which is the kernel in the opposite category. $\lambda_{(n, r)}, \lambda_{0}:(a)_{r}$ tarrows $(b)_{r}$ and $c:(b)_{r} \rightarrow(O)$ such that $c \cdot \lambda_{(n, r)}=O$ and cokernel $c$ is an epimorphism, and in this category the monomorphism is kernel and epimorphism is cokernel, hence the category $\mathscr{R}_{R}$ is an abelian category.

Example 4. Consider $\mathbb{Z}$ the ring of integers and the category $\mathcal{C}$ with objects principal ideals $\{a \mathbb{Z}, a \in \mathbb{Z}\}$ and morphisms $\operatorname{hom}(a \mathbb{Z}, b \mathbb{Z})=$ $\left\{\lambda_{(n, r)}: x \rightarrow n x+r x, n \in \mathbb{Z} ; r \in R\right\} \quad$ is an abelian category.

Example 5. Consider the category $\mathcal{C}$ whose objects are principal right ideals of the ring $M_{2}\left(Z_{2}\right)$, that is the objects are

$$
\begin{gathered}
I_{i}=\left\{\left(\begin{array}{ll}
a & b \\
0 & 0
\end{array}\right), \text { where } a, b \in Z_{2}\right\}, I_{j}= \\
\left\{\left(\begin{array}{ll}
0 & 0 \\
a & b
\end{array}\right), \text { where } a, b \in Z_{2}\right\}
\end{gathered}
$$

and morphisms

$$
\operatorname{hom}\left(I_{i}, I_{j}\right)=\left\{\lambda_{(n, r)}: x \rightarrow n x+r x, n \in Z ; r \in R\right\}
$$


is an abelian category.

Under the partial order $\subseteq$ 'set inclusion', the category $\mathscr{R}_{R}$ is a category with subobjects and has canonical factorization $\lambda_{(n, r)}=m \cdot e \quad$ where $m=\operatorname{ker}\left(\operatorname{coker} \lambda_{(n, r)}\right)$ and $e=\operatorname{coker}\left(\operatorname{ker} \lambda_{(n, r)}\right)$. Dually $\mathcal{L}_{R}$ the category whose objects are principal left ideals of ring $R$ is also an abelian category with subobjects and canonical factorization.

\section{References}

[1] S. Mac Lane (1971) Categories for the working mathematician, Springer Verlag, Newyork, ISBN
0-387-98403-8

[2] Steve Awodey(2006) Category theory, Clarendon Press, Oxford, ISBN 0-19-856861-4 978-0-19-856861-2.

[3] C.Musili (1992) Introduction to rings and modules, Narosa Publishing House, New Delhi, ISBN 81-85198-64-0.

[4] Louis Rowen (1988) Ring theory Vol 1, Academic Press, Inc. New York, ISBN 0-12-599841-4.

[5] Michael Artin (1991) Algebra, Prentice Hall, New Jersey, ISBN 0-13-004763-5.

[6] Mark V Lawson (1998) Inverse semigroups: The theory of partial symmetries, World Scientific, ISBN 978-981-02-3316-7.

[7] Petrq Petro (2002) Green's relations and minimal quasi ideals in rings, Communications in algebra,vol 30, pp. 4677-4686. 